\title{
Hot carrier generation in two-dimensional silver nanoparticle array at different excitation wavelengths under on-resonant condition
}

Yuki Takeuchi, ${ }^{\dagger}$ Antoine Violas, ${ }^{\dagger \dagger}{ }^{\dagger}$ Tetsuya Fujita, ${ }^{\dagger}$ Yasuaki Kumamoto,,$\stackrel{\perp}{ }$

Mircea Modreanu, Takuo Tanaka, $,, \nabla, \uparrow \mid$ Katsumasa Fujita ${ }^{\S, \diamond, \square}$ and Nobuyuki Takeyasu ${ }^{\dagger, *}$

$\dagger$ Graduate School of Natural Science and Technology, Okayama University, 3-1-1,

Tsushima-naka, Kita-ku, Okayama 700-8530, Japan

$¥$ Sorbonne Université - UPMC, Univ. Paris 06, UFR 925, Paris, 75005, France

$\S$ Department of Applied Physics, Graduate School of Engineering, Osaka University, 2-

1, Yamadaoka, Suita, Osaka 565-0871, Japan

${ }^{\perp}$ Department of Pathology and Cell Regulation, Graduate School of Medical Sciences,

Kyoto Prefectural University of Medicine, 465 Kajiicho, Kawaramachi-Hirokoji,

Kamigyo-ku, Kyoto, Kyoto 602-8566, Japan.

I Tyndall National Institute, University College Cork, Lee Maltings, Dyke Parade, Cork, T12 R5CP, Ireland.

\# Metamaterials Laboratory, RIKEN Cluster for Pioneering Research, 2-1, Hirosawa,

Wako, Saitama 351-0198, Japan

$\nabla$ Innovative Photon Manipulation Research Team, RIKEN Center for Advanced Photonics, 2-1, Hirosawa, Wako, Saitama 351-0198, Japan

- Institute of Post-LED Photonics, Tokushima university, 2-1, Minamijosanjima, Tokushima 770-8506, Japan 
${ }^{\circ}$ AIST-Osaka University Advanced Photonics and Biosensing Open Innovation Laboratory, AIST, Suita, Osaka 565-0851, Japan

${ }^{\square}$ Transdimensional Life Imaging Division, Institute for Open and Transdisciplinary Research Initiatives, Suita, Osaka 565-0871, Japan

* Corresponding author: takeyasu@okayama-u.ac.jp, +81-(0)86-251-7845

\section{Simulation model for Discrete dipole approximation}

In the simulations, we hexagonally aligned $30 \mathrm{AgNPs}(40 \mathrm{~nm})$ in yz-plane with the inter-particle gap $(G)$ of 1, 2, 4 and $6 \mathrm{~nm}$, as shown in Fig. 4. In DDA calculation, the metallic structures are assumed to be composed by discrete dipoles. SFig. 1 shows magnified AgNP array $(G=4 \mathrm{~nm})$ composed of dipoles separated by $1 \mathrm{~nm}$. Therefore, the AgNP was composed of 33401 dipoles, which resulted in 1002030 dipoles for the whole AgNP array. The optical response of the AgNP was calculated based on Mie theory, where the incident light is plane wave propagating along the $\mathrm{x}$-axis. We referred the complex dielectric function of silver, which was assumed to be isotropic in $\mathrm{x}, \mathrm{y}$ and $\mathrm{z}$ axes, to Ref. S1.

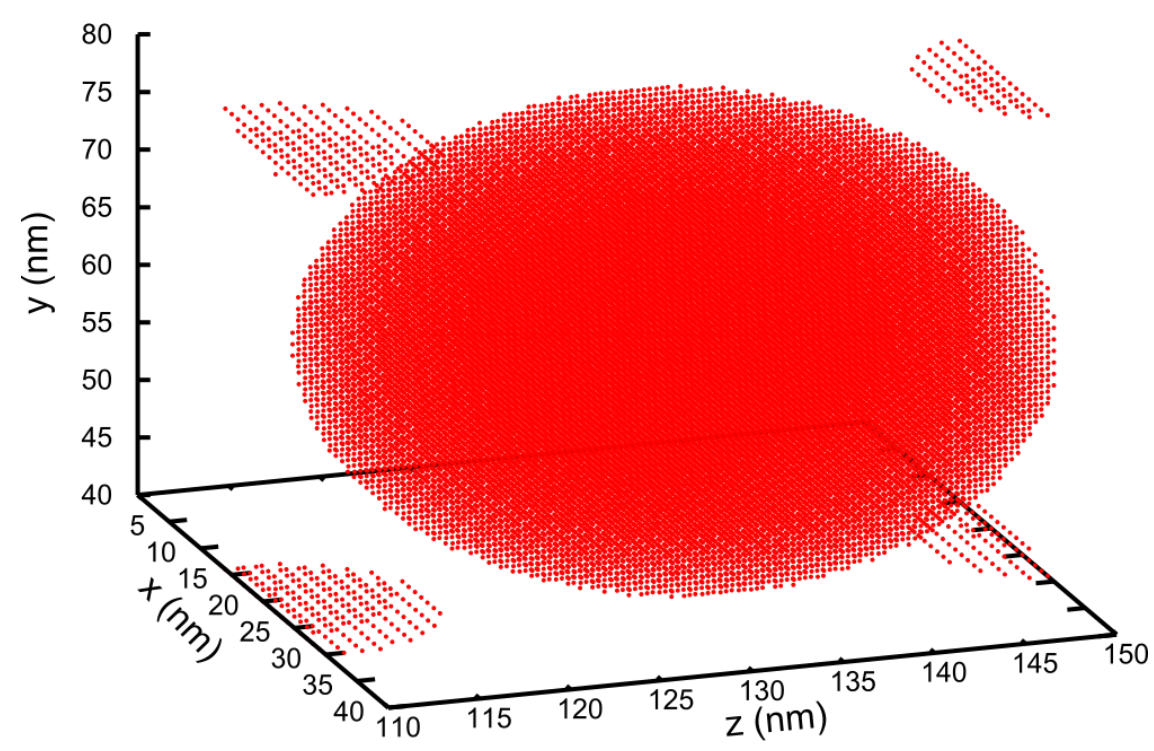

SFigure 1. Simulation model for DDA. AgNPs are composed of dots, which represent dipoles.

\section{Absorption spectrum of $p$-ATP}

Absorption spectrum of $p$-ATP was measured, which is shown in SFig. 2. Almost no absorption was observed at $>450 \mathrm{~nm}$ although ultraviolet light is strongly absorbed. 


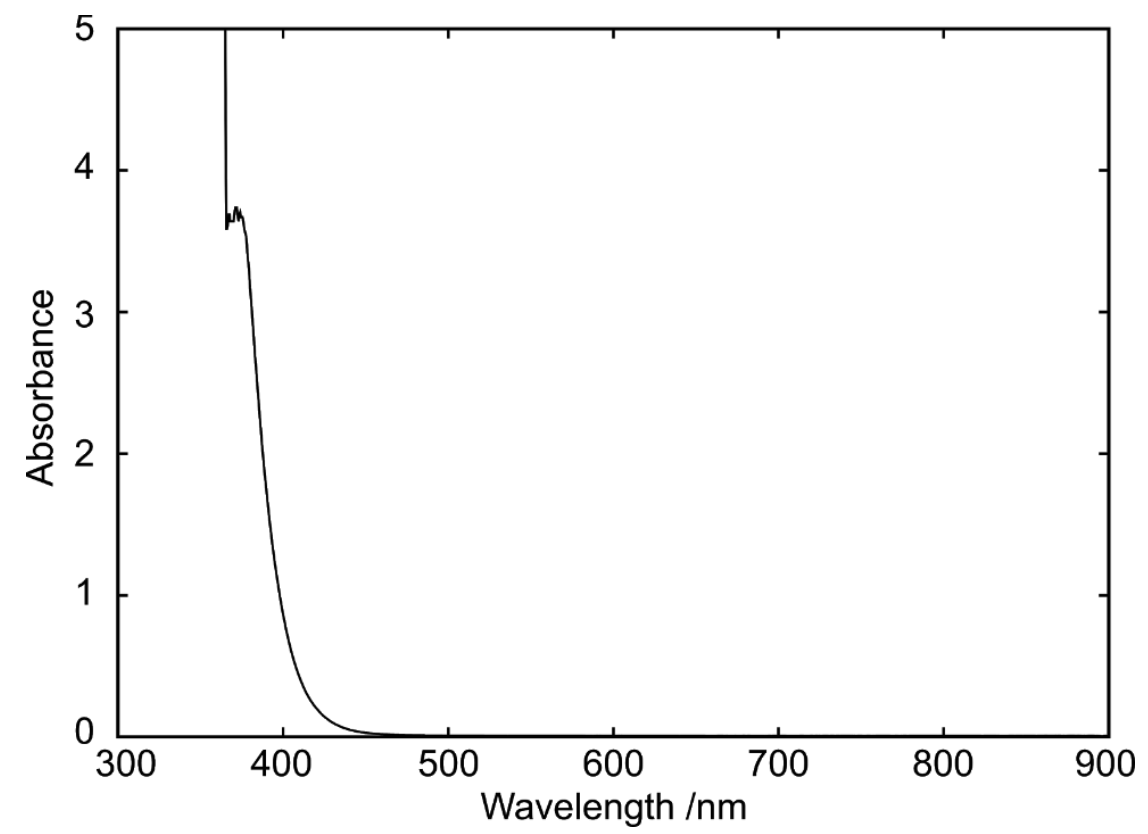

SFigure 2. Absorption spectrum of $p$-ATP (c.a. $1 \mathrm{wt} \%$ in ethanol).

\section{The comparison of peak width at $1080 \mathrm{~cm}^{-1}$ between $532 \mathrm{~nm}$ and $458 \mathrm{~nm}$}

We compared the peak width at $1080 \mathrm{~cm}^{-1}$ at the same height between $532 \mathrm{~nm}$ and 458 $\mathrm{nm}$.

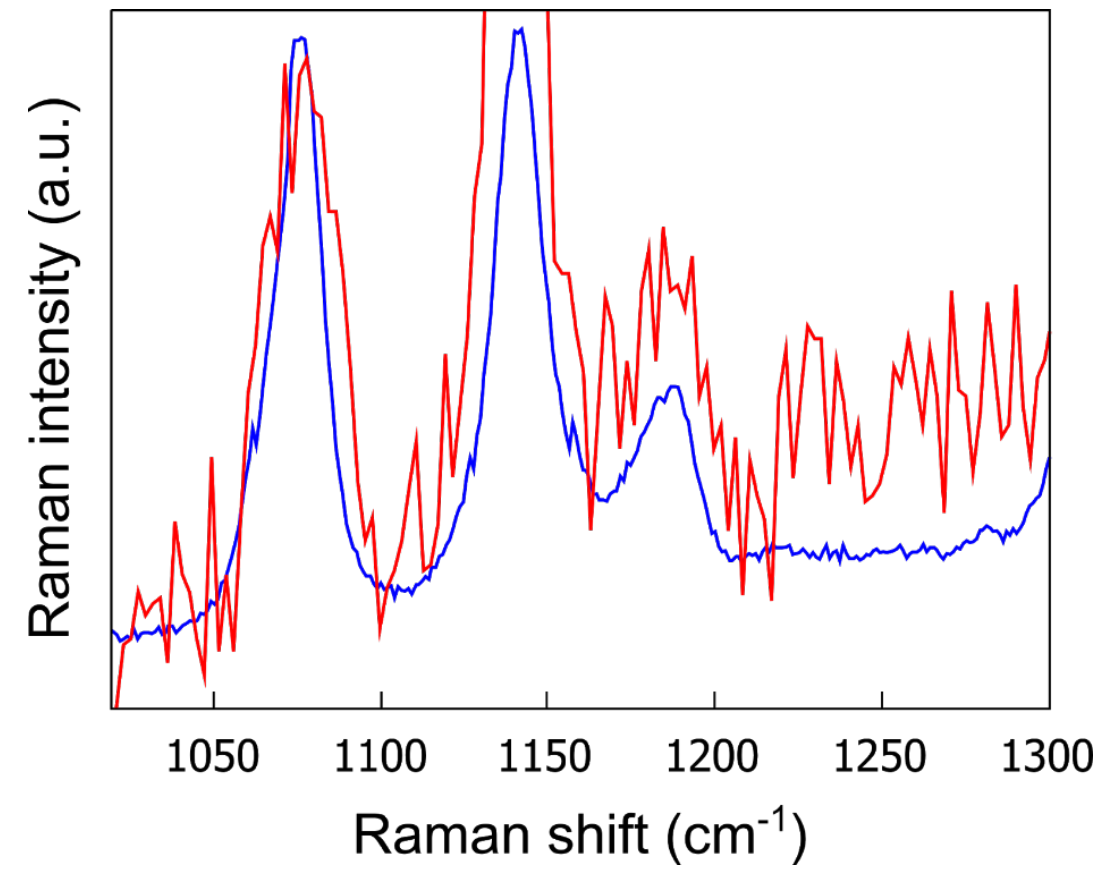

SFigure 3. Comparison of peak width between $532 \mathrm{~nm}$ (blue) and $458 \mathrm{~nm}$ (red) 


\section{Absorption and Scattering components in Extinction of AgNP arrays}

We calculated the extinction spectra of AgNP arrays at the inter-particle gaps of 6, 4, 2 and $1 \mathrm{~nm}$ with DDA, which are shown in Fig. 4. Extinction includes the absorption and scattering components, and the components can be calculated individually in DDA. SFigure 3 shows the absorption and scattering spectra of AgNP arrays at the interparticle gaps of $6,4,2$ and $1 \mathrm{~nm}$, respectively.
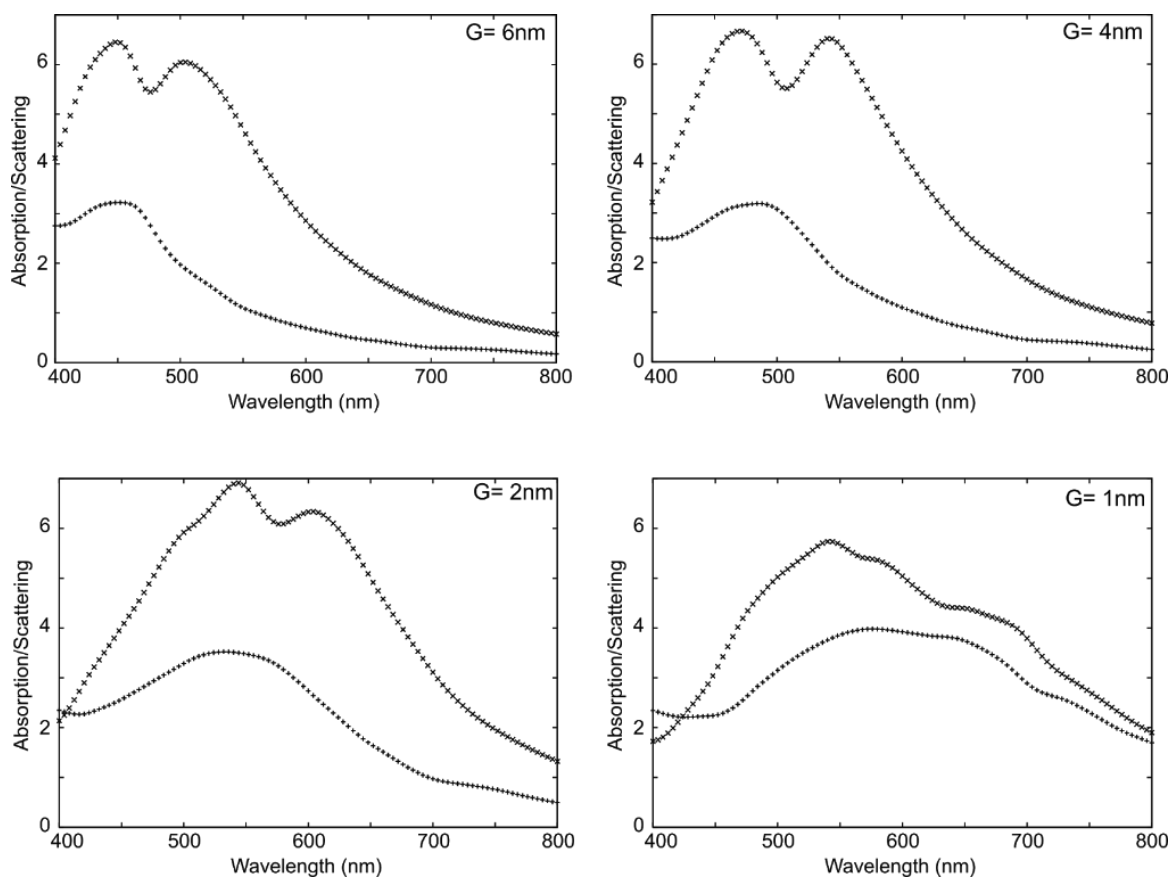

SFigure 4. Absorption (+) and Scattering (x) spectra of AgNP (40 nm) arrays at the inter-particle gaps of 6, 4, 2 and $1 \mathrm{~nm}$.

\section{References}

(S1) A. D. Rakić, A. B. Djurišić, J. M. Elazar, M. L. Majewski, Optical properties of metallic films for vertical-cavity optoelectronic devices. Appl. Opt., 1998, 37, 52715283. 